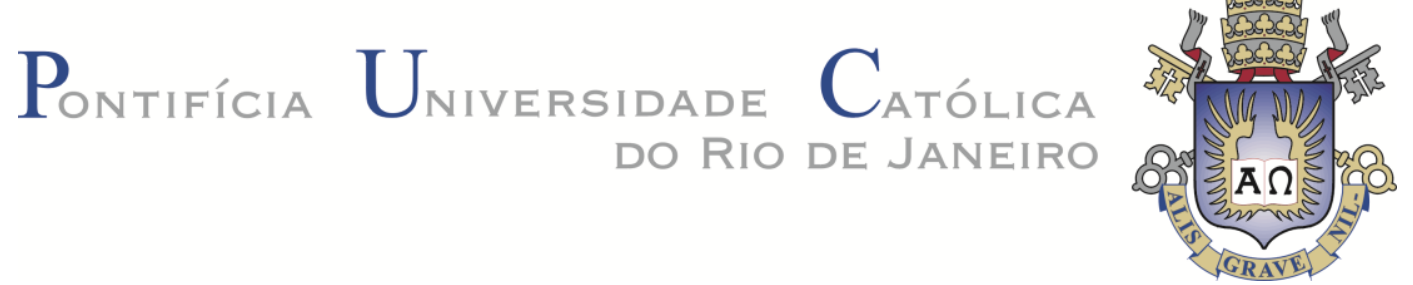

Rogerio do Nascimento Rebello Filho

\title{
Transmissão Óptica de Dados a 50 Gbit/s e eficiência espectral de $1 \mathrm{bit} / \mathrm{s} / \mathrm{Hz}$
}

Dissertação de Mestrado

Dissertação apresentada como requisito parcial para obtenção do título de Mestre pelo Programa de Pós-Graduação em Engenharia Elétrica da PUC-Rio.

Orientador: Jean Pierre von der Weid Co-orientador: Giancarlo Vilela de Faria 
Rogerio do Nascimento Rebello Filho

\title{
Transmissão Óptica de Dados a 50 Gbit/s e eficiência espectral de $1 \mathrm{bit} / \mathrm{s} / \mathrm{Hz}$
}

\begin{abstract}
Dissertação apresentada como requisito parcial para obtenção do título de Mestre pelo Programa de PósGraduação em Engenharia Elétrica da PUC-Rio. Aprovada pela Comissão Examinadora abaixo assinada.
\end{abstract}

Prof. Jean Pierre von der Weid

Orientador

Pontifícia Universidade Católica do Rio de Janeiro

Prof. Giancarlo Vilela de Faria

Co-orientador

Pontifícia Universidade Católica do Rio de Janeiro

Prof. Rodolfo Araújo de Azevedo Lima

IPqM - Instituto de Pesquisa da Marinha

Prof. Evandro Conforti

UNICAMP - Faculdade de Engenharia Elétrica e Computação

Prof. Guilherme Penello Temporão

Pontifícia Universidade Católica do Rio de Janeiro

Prof. José Eugênio Leal

Coordenador Setorial do Centro Técnico Científico PUC-Rio

Rio de Janeiro, 11 de julho de 2013 
Todos os direitos reservados. É proibida a reprodução total ou parcial do trabalho sem autorização da universidade, do autor e do orientador.

\section{Rogerio do Nascimento Rebello Filho}

Graduou-se na Pontifícia Universidade Católica do Rio de Janeiro no segundo semestre de 2010. Seus atuais interesses de pesquisa são na área de comunicação óptica.

Ficha Catalográfica

Rebello Filho, Rogerio do Nascimento

Transmissão Óptica de Dados a $50 \mathrm{Gbit} / \mathrm{s}$ e eficiência espectral de $1 \mathrm{bit} / \mathrm{s} / \mathrm{Hz}$ / Rogerio do Nascimento Rebello Filho ; orientador: Jean Pierre von der Weid ; co-orientador: Giancarlo Vilela de Faria2013.

67 f. : il. (color.) ; $30 \mathrm{~cm}$

Dissertação (mestrado)-Pontifícia Universidade Católica do Rio de Janeiro, Departamento de Engenharia Elétrica, 2013.

Inclui bibliografia

1. Engenharia elétrica - Teses. 2. Comunicação Óptica. 3. Multiplexação por Divisão do Comprimento de Onda. 4. Multiplexação em Polarização. 5. Modulação ASK. 6. Modulação DQPSK 7. Eficiência Espectral. I. von der Weid, Jean Pierre. II. de Faria, Giancarlo Vilela. III. Pontifícia Universidade Católica do Rio de Janeiro. Departamento de Engenharia Elétrica. IV. Título. 


\section{Agradecimentos}

Ao prof. Jean Pierre von der Weid pela oportunidade que me foi concedida.

Ao meu co-orientador que sempre esteve presente em todos os casos disposto a fornecer auxílio, que além de um respeito profissional, tenho uma admiração na forma de companheirismo demonstrado em todos os momentos. Muito obrigado, Giancarlo Vilela de Faria.

Ao doutor Rodolfo Lima pelo apoio na fase final.

Ao doutor Tiago Ferreira pela sua simplicidade e competência nas discussões sobre o meu trabalho.

À minha irmã e irmão pelo apoio e amparo em diversas situações.

Aos meus pais que me ajudaram a chegar até aqui.

À minha bisavó pelo apoio durante meu período da faculdade.

Aos demais amigos da PUC-Rio pelo companheirismo nesses anos.

A todos os funcionários e professores do CETUC, pelos ensinamentos adquiridos durante este período.

Ao CNPq, pelo apoio financeiro.

E a todos aqueles que de alguma maneira tenham contribuído para a realização deste trabalho. 


\section{Resumo}

Rebello Filho, Rogerio do Nascimento; von der Weid, Jean Pierre (Orientador); de Faria, Giancarlo Vilela (Co-orientador). Transmissão Óptica de Dados a $50 \mathrm{Gbit} / \mathrm{s}$ e eficiência espectral de $1 \mathrm{bit} / \mathrm{s} / \mathrm{Hz}$. Rio de Janeiro, 2013. 67p. Dissertação de Mestrado - Departamento de Engenharia Elétrica, Pontifícia Universidade Católica do Rio de Janeiro.

Neste trabalho realizamos uma prova da viabilidade de um sistema de comunicação óptica com capacidade de transmissão de 50 Gbit/s em uma largura de banda de $50 \mathrm{GHz}$ utilizando o legado dos sistemas com taxas de $10 \mathrm{Gbit} / \mathrm{s}$. Uma série de configurações experimentais foi testada em uma ordem de complexidade crescente para verificar separadamente as etapas e as técnicas aplicadas para o aumento da capacidade de transmissão de dados e a eficiência espectral. Em alguns casos, a curva característica resultante da configuração backto-back do analisador de taxa de erro de bit média foi utilizada como referência para comparação das configurações experimentais realizadas durante o trabalho.

\section{Palavras-Chave}

Comunicação Óptica; Multiplexação por Divisão do Comprimento de Onda; Multiplexação em Polarização; Modulação ASK; Modulação DQPSK; Eficiência Espectral. 


\section{Abstract}

Rebello Filho, Rogerio do Nascimento; von der Weid, Jean Pierre (Advisor); de Faria, Giancarlo Vilela (Co-Advisor). Optical Data Transmission at $50 \mathrm{Gbit} / \mathrm{s}$ and spectral efficiency of $1 \mathrm{bit} / \mathrm{s} / \mathrm{Hz}$. Rio de Janeiro, 2013. 67p. Msc. Dissertation - Departamento de Engenharia Elétrica, Pontifícia Universidade Católica do Rio de Janeiro.

In this work we perform a proof of feasibility of $50 \mathrm{Gbit} / \mathrm{s}$ transmission within a $50 \mathrm{GHz}$ optical bandwidth exploring the $10 \mathrm{Gbit} / \mathrm{s}$ legacy. A series of experimental configurations were tested in an order of increasing complexity to verify separately the steps and applied techniques for increasing data transmission capacity and spectral efficiency. In some cases, the comparison of experimental configuration was made using the back-to-back configuration of the Bit Error Rate Tester.

\section{Keywords}

Optical Communication; Wavelength Division Multiplexing; Polarization Multiplexing; ASK Modulation; DQPSK Modulation; Spectral Efficient. 


\section{Sumário}

1 Introdução

1.1. Sistema Básico de Comunicação Óptica utilizando

Fibra Óptica

1.2. Motivação da utilização de Sistemas de Comunicação

Óptica

2 Comunicação Óptica: Apresentação e Análise das

Tecnologias mais Relevantes na Atualidade

2.1. O Transmissor Óptico e Métodos para

Incremento da Capacidade e/ou Eficiência Espectral

2.1.1. Discussão sobre os Principais Formatos de

Modulação Empregados

2.1.2. Discussão sobre os Principais Métodos de

Multiplexação Empregados

2.2. O Receptor Óptico

3 Descrição das Técnicas Implementadas nas Configurações

Experimentais 24

3.1. Multiplexação por Divisão de Comprimento de Onda (WDM) 24

3.2. Multiplexação em Polarização (PolMUX) 28

3.3. O Modulador 30

3.3.1. Modulador Mach-Zehnder 30

3.3.2. Modulação NRZ-ASK e VSB NRZ-ASK 33

4 Experimentos e Resultados 34

4.1. Curva de Referência: Configuração Back-to-back 34

4.2. Análise do Desempenho do Sistema sob Filtragem em DWDM 36

4.2.1. Análise de desempenho com o centro do filtro

deslocado da frequência da portadora óptica 
4.3. Avaliação do efeito da Multiplexação Densa por Divisão de Comprimento de Onda 44

4.4. Avaliação do Efeito da Multiplexação em Polarização 49

4.5. Multiplexação Densa por Divisão em Comprimento de Onda e em conjunto com Multiplexação em Polarização 52

4.6. Geração de Múltiplas Portadoras 56

5 Conclusões

6 Referências Bibliográficas 66 


\section{Lista de Figuras}

Figura 1 - Diagrama em blocos dos elementos básicos de um

Sistema de Comunicação Óptica

Figura 2 - (a) Esquema de um sistema de comunicação com canal único

ponto a ponto (b) Implementação de um sistema WDM

Figura 3 - Atenuação de uma fibra monomodo simples em função

do comprimento de onda

Figura 4 - Esquema básico de multiplexação em polarização

Figura 5 - Diagrama em blocos do Controlador Automático de Polarização

Figura 6 - Diagrama Funcional do modulador Mach-Zehnder

Figura 7 - Estrutura de um modulador Mach-Zehnder

Figura 8 - Modulação no formato NRZ, (a) sinal modulante (b) sinal com modulação ASK

Figura 9 - Configuração Experimental para determinação da Curva de

Referência

Figura 10 - Curva BER x Potência de entrada do receptor $[\mathrm{dBm}]$ na configuração back-to-back

Figura 11 - Configuração Experimental para análise da filtragem para técnica DWDM

Figura 12 - Penalidade $[\mathrm{dB}]$ x Espaçamento entre canais $[\mathrm{GHz}]$ com

filtro centrado na portadora

Figura 13 - Diagrama de Olho para espaçamento entre Canais de $25 \mathrm{GHz}$

na configuração apresentada na Figura 11

Figura 14 - Digrama de Olho para espaçamento entre canais de $15 \mathrm{GHz}$

Figura 15 - Diversos espectros do sinal na entrada do receptor para uma

taxa de erro de bit de 1E-9 deslocando a portadora em relação ao centro

do filtro

Figura 16 - Resultado da simulação da penalidade em função do

deslocamento da frequência da portadora para uma BER =1E-9 
Figura 17 - Configuração Experimental para análise da técnica de

Multiplexação em Comprimento de Onda em sistema DWDM

Figura 18 - Espectro dos cinco canais na entrada do amplificador

óptico EDFA

Figura 19 - BER x Potência na entrada do receptor [dBm] 47

Figura 20 - BER x OSNR [dB] 49

Figura 21 - Configuração Experimental para Avaliação da Multiplexação em Polarização

Figura 22 - Curva BER para uma transmissão com um único canal e para uma transmissão usando 2 canais de 10GBit/s utilizando a técnica de multiplexação em polarização

Figura 23 - 50 Gbit/s utilizando POLMUX em conjunto com um sistema UDWDM de $50 \mathrm{GHz}$

Figura 24 - Espectros dos 3 canais polarizados verticalmente e espectro pontilhado dos dois canais restantes com polarização ortogonal

Figura 25 - Espectro dos cinco canais de comunicação aquisitados na saída do módulo transmissor

Figura 26 - (a) Espectro dos canais com centrados no máximo do filtro utilizado para e demultiplexação e (b) o mesmo espectro com a frequência deslocada em relação ao valor de máximo do filtro

Figura 27 - Sinal de realimentação utilizado para alimentar o controlador automático de polarização

Figura 28 - Geração de Múltiplas Portadoras

Figura 29 - Espectro do sinal na saída do modulador Mach-Zehnder simples de geração de múltiplas portadoras

Figura 30 - Configuração experimental para verificação do desempenho do gerador de multiportadoras

Figura 31 - Espectro dos sinais modulados utilizando a técnica de geração de multiportadoras

Figura 32 - Espectro do sinal antes do segundo demulplexador com o canal central desligado

Figura 33 - Espectro do canal central após a filtragem realizada pelo segundo demultiplexador

Figura 34 - Desempenho da configuração para geração de multiportadoras 
Figura 35 - Taxa média de erro de bits média x OSNR [dB] correspondente ao canal central 


\section{Lista de Abreviações}

ASE - Emissão Espontânea Amplificada (Amplified Spontaneous Emission)

ASK - Chaveamento de Amplitude (Amplitude Shift Keying)

BER - Taxa de Erro de Bits (Bit Error Ratio)

CETUC - Centro de Estudos em Telecomunicação da PUC-Rio

CD - Dispersão Cromática (Chromatic Dispersion)

CDM - Multiplexação por Divisão de Código (Code Division Multiplexing)

CW - Onda Contínua (Continuous Wave)

DEMUX - Demultiplexador (Demultiplexer)

DQPSK - Modulação diferencial por chaveamento de fase em quadratura

(Differential Quadrature Phase Shift)

DWDM - Multiplexagem Densa por Divisão de Comprimento de Onda

(Dense Wavelenght Division Multiplexing)

DSP - Processadores de Sinais Digitais (Digital Signal Processsors)

DBPSK - Modulação diferencial binária por chaveamento de fase (Differential Binary Phase Shift Keying)

EDFA - Amplificador a Fibra Dopada com Érbio (Erbium Doped Fiber Amplifier)

FEC - Códigos Corretores de Erros (Forward Error Control)

FPGA - (Field-Programmable Gate Array)

LED - Diodo Emissor de Luz (Light Emitting Diode)

LNA - Amplificador de Baixo Ruído (Low Noise Amplifier)

MZM - Modulador Mach-Zehnder (Mach-Zehnder Modulator)

MUX - Multiplexador (Multiplex)

NRZ - Não Retorna a Zero (Non-Return-to-Zero)

OOK - Chaveamento Liga-Desliga (On-Off Keying)

OSA - Analisador de Espectro Óptico (Optical Spectrum Analyzer)

OSNR - Relação Sinal-Ruído Óptico (Optical Signal-to-Noise Ratio)

PMD - Dispersão dos Modos de Polarização (Polarization Mode

Dispersion)

PRBS - Sequência Binária Pseudo-Aleatória (Pseudo-Random Binary Sequence) 
RF - Rádio Frequência (Radio Frequency)

RX - Receptor (Reception)

RZ - Retorno ao Zero (Return-to-Zero)

SDM - Multiplexação por Divisão do Espaço (Space Division Multiplexing)

TX - Transmissor (Transmission)

TDM - Multiplexação por Divisão do Tempo (Time Division Multiplexing)

VSB - Banda Lateral Vestigial (Vestigial Sideband)

WDM - Multiplexação por Divisão de Comprimento de Onda (Wavelength Division Multiplex) 